\title{
SÍNTESE DE REDES FLEXÍVEIS DE TROCADORES DE CALOR VIA PROGRAMAÇÃO MATEMÁTICA UTILIZANDO UM MÉTODO DE OTIMIZAÇÃO SIMULTÂNEO
}

\author{
C. B. MIRANDA ${ }^{1}$, M. A. S. S. RAVAGNANI ${ }^{1}$ \\ ${ }^{1}$ Universidade Estadual de Maringá, Departamento de Engenharia Química \\ E-mail para contato: camila.mir4@gmail.com
}

\begin{abstract}
RESUMO - As indústrias, muitas vezes, necessitam de redes de trocadores de calor (RTC) que suportam variações nas capacidades térmicas e nas temperaturas das correntes em um número finito de períodos de operação. Este trabalho apresenta um método simultâneo, no qual todas as variáveis são otimizadas ao mesmo tempo, via programação matemática para a síntese de RTC flexíveis. O modelo utiliza uma superestrutura que prevê a divisão de correntes e é formulado como um problema de programação não linear mista inteira (PNLMI). O modelo realiza um trade-off entre os custos de capital e operacional e deve ser aplicado para cada período de operação separadamente. Para integrar as estruturas alcançadas, desenvolveu-se um procedimento computacional com a finalidade de realizar esta tarefa automaticamente. Um exemplo da literatura foi utilizado com o objetivo de comparar os resultados encontrados entre o modelo desenvolvido no presente trabalho e os apresentados na literatura. Os resultados para os custos totais encontrados foram menores que os da literatura.
\end{abstract}

\section{INTRODUÇÃO}

Para atender as solicitações das indústrias, muitas vezes, é necessário desenvolver RTC flexíveis, ou seja, redes que suportam variações nas condições de operação em um número finito de períodos de operação (JIANG; CHANG, 2013). A programação matemática pode ser utilizada na síntese de RTC, pois este tipo de problema pode ser formulado como um problema de otimização. Os métodos de otimização simultâneos podem atingir resultados melhores que os alcançados pelos métodos sequenciais, porque sintetizam a rede sem a decomposição do problema. (RAVAGNANI; CABALLERO SUÁREZ, 2012).

Alguns autores desenvolveram algoritmos utilizando a programação matemática. Yee e Grossmann (1990) apresentaram um modelo de PNLMI para síntese de RTC com condições fixas de operação, em que o custo de utilidades, a área dos trocadores e a seleção das trocas são otimizados simultaneamente. $O$ modelo utiliza uma superestrutura baseada na representação por estágios divididos por temperaturas, em que dentro de cada estágio existe a possibilidade de cada corrente quente trocar calor com todas as correntes frias e vice-versa, e supõe a mistura isotérmica para as correntes que apresentam divisão de vazão. Aaltola (2002) desenvolveu um modelo simultâneo com o propósito de gerar uma RTC flexível em uma única etapa. O modelo foi baseado no trabalho de Yee e Grossmann (1990), em que a área da troca térmica considerada na função objetivo é calculada pela média dos valores das áreas 
dessa troca nos diferentes períodos. Chen, Kuo e Hung (2005) apresentaram um modelo simultâneo para a síntese de RTC que possui distúrbios esperados nas temperaturas e nas vazões das correntes decomposto em quatro etapas principais e omitiram a hipótese de mistura isotérmica.

Verheyen e Zhang (2006) modificaram o modelo de PNLMI proposto por Aaltola (2002), utilizaram a área máxima entre os períodos para cada unidade na função objetivo e incluíram um modelo de PNL para melhoria da solução. Isafiade e Fraser (2010) desenvolveram um modelo para RTC multiperiódicas baseado na superestrutura IBMS (interval-based MINLP superstructure), a qual permite períodos com duração desiguais determinados antecipadamente para serem incorporados na formulação do modelo. Jiang e Chang (2013) desenvolveram um projeto com o objetivo de ajustar os comprimentos dos períodos em resposta às perturbações inesperadas durante operações reais; de contornar os desperdícios nas capacidades de trocadores de calor que possuem mudanças significantes em períodos diferentes e de diminuir a complexidade dos modelos simultâneos. Aplica o modelo de PNLMI para produzir o ótimo para cada período separadamente e utiliza uma estratégia de partilha de tempo para integrar as estruturas de modo a reduzir o custo de investimento.

Assim, o presente trabalho propõe um modelo de otimização simultâneo, baseado na superestrutura de Yee e Grossman (1990) para alcançar uma RTC flexível via programação matemática. $\mathrm{O}$ modelo realiza o balanço de energia com a temperatura de saída do trocador e não com a temperatura do intervalo e utiliza equação de igualdade para calcular as diferenças de temperaturas dos terminais das unidades de troca térmica. Aplica-se o modelo para cada período de operação separadamente e para integrar as estruturas alcançadas, utiliza-se de um algoritmo que gera automaticamente as áreas que serão atribuídas, a fim de reduzir o custo de investimento e manter as taxas de consumo de utilidades em cada período a níveis mínimos.

\section{MODELO}

O modelo de PNLMI deve ser aplicado para cada período de operação separadamente e alguns dados precisam ser conhecidos: temperatura de entrada e saída da corrente quente e fria e da utilidade quente e fria $\left(\right.$ Thin $_{i}$, Thout $_{i}$, Tcin $_{j}$, Tcout $_{j}$, Tsin $_{m}$, Tsout $_{m}$, Twin $_{n}$ e Twout $\left.{ }_{n}\right)$; capacidade térmica da corrente quente e fria $\left(F h_{i}\right.$ e $\left.F c_{j}\right)$; custo das utilidades quente e fria $\left(C_{m}\right.$ e $\left.C_{n}\right)$; coeficiente global de transmissão de calor entre os pares $(i, j),(m, j)$ e $(i, n)\left(\operatorname{Co}_{i, j}, \operatorname{Cos}_{m, j}\right.$ e $\left.\operatorname{Cow}_{i, n}\right)$; limite superior para a diferença de temperatura nas unidades $\left(m t_{i, j}, \quad m t s_{m, j}\right.$ e $\left.m t w_{i, n}\right)$; limite superior do calor trocado entre os pares $\left(m q_{i, j, k}\right.$, $m q s_{m, j}$ e $\left.m q w_{i, n}\right)$; coeficientes da equação do custo de investimento $(a, b, c)$; fator de anualização $(r)$; e temperatura mínima de aproximação (EMAT), em que os índices $i$ ou $i i$ representam a corrente quente, $j$ ou $j j$ representam a corrente fria, $m$ e $n$ representam as utilidades quente e fria e $k$ representa os estágios que são divididos em intervalos de temperatura. O conjunto de estágios da superestrutura é indicado por $S T$, em que $N O K$ indica o número total de estágios.

As variáveis são: diferença de temperatura nos terminais quente e frio do trocador $(i, j)$ $\left(d t h_{i, j, k}\right.$ e $\left.d t c_{i, j, k}\right)$; diferença de temperatura no terminal frio do trocador $(m, j)\left(d t s_{m, j}\right)$; diferença de temperatura no terminal quente do trocador $(i, n)\left(d t w_{i, n}\right)$; calor trocado entre $(i, j),(m, j)$ e $(i, n)\left(q_{i, j, k}, q s_{m, j}\right.$ e $\left.q w_{i, n}\right)$; temperatura da corrente quente e fria no intervalo 
$\left(t h_{i, k}\right.$ e $\left.t c_{j, k}\right)$; temperatura de saída da corrente quente e fria no trocador $\left(t h s_{i, j, k}\right.$ e $\left.t c s_{i, j, k}\right)$; proporção de divisão das correntes quente e fria no trocador $\left(r h_{i, j, k}\right.$ e $\left.r c_{i, j, k}\right)$; área do trocador $\left(\operatorname{area}_{i, j, k}, \operatorname{areas}_{m, j}\right.$ e $\left.\operatorname{areaw}_{i, n}\right)$; média logarítmica das diferenças de temperaturas $\left(d t m l_{i, j, k}\right.$, $d t m l s_{m, j}$ e $d t m l w_{i, n}$ ); e custo do trocador (custohc $c_{i, j, k}$, custos $_{m, j}$ e custow $_{i, n}$ ). As variáveis binárias são $y_{i, j, k}, y s_{m, j}$ e $y w_{i n}$ e indicam a existência dos trocadores.

A formulação matemática possui como função objetivo minimizar o custo total da rede anualizado (CTA) e não utiliza a suposição de mistura isotérmica, sendo necessário adicionar balanços de energia nos pontos de mistura. Algumas restrições são apresentadas apenas para as correntes quentes, porém as mesmas devem ser geradas para as correntes frias ou para utilidades:

$$
\begin{gathered}
\min \mathrm{CTA}=\sum_{i} \sum_{j} \sum_{k \in S T} \text { custoh }_{i, j, k}+\sum_{m} \sum_{j} \operatorname{custos}_{m, j}+\sum_{i} \sum_{n} \text { custow }_{i, n} \\
+\left(\sum_{m} \sum_{j} C_{m} \cdot q s_{m, j}\right)+\left(\sum_{i} \sum_{n} C_{n} \cdot q w_{i, n}\right)
\end{gathered}
$$

Sujeito a:

- Balanço de energia global para cada corrente quente e fria:

$$
\left(\operatorname{Thin}_{i}-\text { Thout }_{i}\right) \cdot F h_{i}=\left(\sum_{k \in S T} \sum_{j} q_{i, j, k}\right)+\left(\sum_{n} q w_{i, n}\right)
$$

- Balanço de energia em cada etapa da superestrutura para corrente quente e fria:

$$
\left(t h_{i, k}-t h_{i, k+1}\right) \cdot F h_{i}=\sum_{j} q_{i, j, k} \quad \forall k \in S T
$$

- Balanço de energia para cada trocador:

$$
\begin{aligned}
& \left(t h_{i, k}-t h s_{i, j, k}\right) \cdot r h_{i, j, k} \cdot F h_{i}=q_{i, j, k} \quad \forall k \in S T \\
& \left(t c s_{i, j, k}-t c_{j, k+1}\right) \cdot r c_{i, j, k} \cdot F c_{j}=q_{i, j, k} \quad \forall k \in S T
\end{aligned}
$$

- Somatório da proporção de divisão das correntes quente e fria em cada etapa:

$$
\sum_{j} r h_{i, j, k}=1 \quad \forall k \in S T
$$

- Temperatura de saída de cada trocador:

$$
\sum_{j}\left(r h_{i, j, k} \cdot t h s_{i, j, k}\right)=t h_{i, k+1} \quad \forall k \in S T
$$




$$
\sum_{i}\left(r c_{i, j, k} \cdot t c s_{i, j, k}\right)=t c_{j, k} \quad \forall k \in S T
$$

- Viabilidade das temperaturas: a temperatura da corrente quente na primeira etapa deve ser igual a temperatura de entrada dessa corrente; a temperatura da corrente fria no último intervalo de temperatura deve ser igual a temperatura de entrada dessa corrente; a temperatura das correntes quente e fria no intervalo de temperatura inferior deve ser maior ou igual a temperatura dessa corrente do intervalo superior; a temperatura da corrente quente no último intervalo de temperatura deve ser maior ou igual a temperatura de saída dessa corrente; e a temperatura da corrente fria na primeira etapa estágio deve ser menor ou igual a temperatura de saída dessa corrente.

- Cargas das utilidades quente e frias:

$$
\left(t h_{i, N O K+1}-\text { Thout }_{i}\right) \cdot F h_{i}=\sum_{n} q w_{i, n}
$$

- Restrições lógicas para as correntes de processo e utilidades

$$
q_{i, j, k} \leq y_{i, j, k} \cdot m q_{i, j, k} \quad \forall k \in S T
$$

- Cálculo das temperaturas de aproximação:

$$
\begin{aligned}
& d t h_{i, j, k}=t h_{i, k}-t c s_{i, j, k}+m t_{i, j} \cdot\left(1-y_{i, j, k}\right) \quad \forall k \in S T \\
& d t c_{i, j, k}=t h s_{i, j, k}-t c_{j, k+1}+m t_{i, j} \cdot\left(1-y_{i, j, k}\right) \quad \forall k \in S T \\
& d t s_{m, j}=\text { Tsout }_{m}-t c_{j, 1}+m t s_{m, j} \cdot\left(1-y s_{m, j}\right) \\
& d t w_{i, n}=t h_{i, N O K+1}-\text { Twout }_{n}+m t w_{i, n} \cdot\left(1-y w_{i, n}\right)
\end{aligned}
$$

- Cálculo das áreas das unidades que trocam calor entre correntes de processo e entre correntes e utilidades, em que as médias logarítmicas das diferenças de temperaturas são calculadas pela aproximação de Chen (1987):

$$
q_{i, j, k}=\mathrm{Co}_{i, j} \cdot \operatorname{area}_{i, j, k} \cdot d t m l_{i, j, k} \quad \forall k \in S T
$$

- Cálculo dos custos dos trocadores:

$$
\begin{aligned}
& \text { custohc }_{i, j, k}=r \cdot a \cdot y_{i, j, k}+r \cdot b \cdot\left(\text { area }_{i, j, k}\right)^{c} \quad \forall k \in S T \\
& \text { custos }_{m, j}=r \cdot a \cdot y s_{m, j}+r \cdot b \cdot\left(\text { areas }_{m, j}\right)^{c} \\
& \text { custow }_{i, n}=r \cdot a \cdot y w_{i, n}+r \cdot b \cdot\left(\text { areaw }_{i, n}\right)^{c}
\end{aligned}
$$

Após resolver esse modelo para cada período de operação, deve-se realizar um procedimento para integrar as estruturas alcançadas e reduzir o custo de investimento. $\mathrm{O}$ programa foi desenvolvido no ambiente Matlab e o algoritmo é apresentado na Figura 1. 


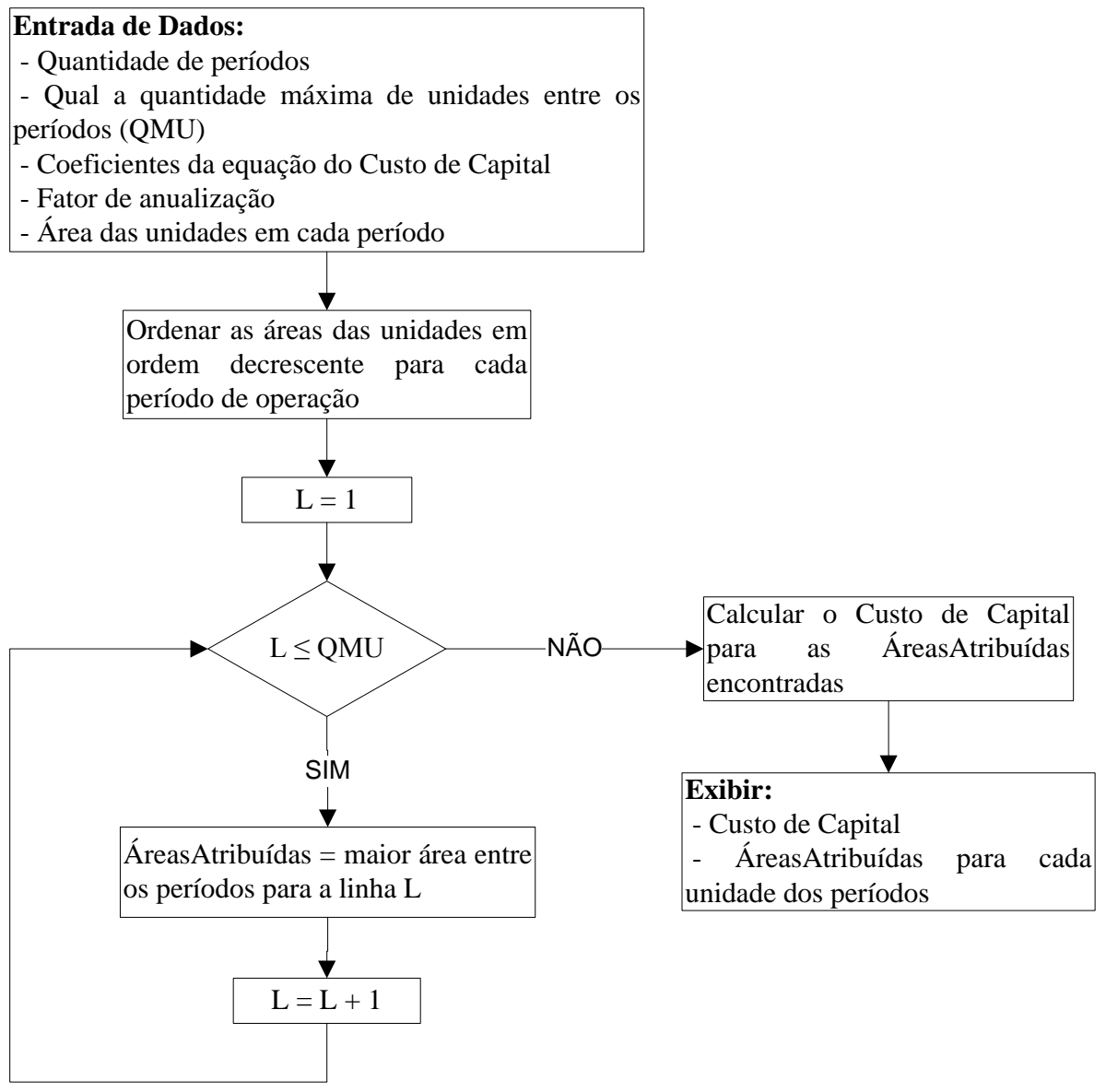

Figura 1 - Algoritmo para a integração das estruturas.

Cada unidade de trocada térmica é projetada com o valor da área atribuída. Para evitar contaminação entre as diferentes correntes que podem passar em um mesmo tubo e em uma mesma válvula, assume-se que entre cada período será realizada uma limpeza completa nas tubulações. Este procedimento deve ocorrer, porque por um mesmo trocador de calor pode passar correntes diferentes em períodos diferentes.

\section{ESTUDO DE CASO}

O exemplo, resolvido por Jiang e Chang (2013), apresenta duas correntes quentes, duas correntes frias, uma utilidade quente $(H U)$ e uma utilidade fria $(C U)$, em que é necessário operar em três períodos. As condições de operação sofrem alterações de um período para o outro e são exibidas na Tabela 1. Para a utilidade quente, a temperatura de entrada e saída é $680 \mathrm{~K}$, o coeficiente individual de transferência de calor é $5 \mathrm{~kW} /\left(\mathrm{m}^{2} \mathrm{~K}\right)$ e o custo é $150,163 \$ /(\mathrm{kW} \cdot$ ano). Já para a água de resfriamento, as temperaturas são $300 \mathrm{~K}$ e $320 \mathrm{~K}$, respectivamente, o coeficiente individual de troca térmica é $1 \mathrm{~kW} /\left(\mathrm{m}^{2} \mathrm{~K}\right)$ e o custo é $53,064 \$ /(\mathrm{kW} \cdot \mathrm{ano})$. Além disso, adota-se: Custo $=4333 \cdot$ Area ${ }^{0,6}$, Area [=] $\mathrm{m}^{2}$; $E M A T=10 \mathrm{~K}, r=0,1$. O modelo de PNLMI foi resolvido separadamente para cada período de operação na ferramenta de otimização GAMS, com a versão $22.6 \mathrm{em}$ um processador Intel Core 5, 2.60 GHz. Utilizou-se o solver DICOPT e o tempo de processamento foi menor que 1 segundo em todos os períodos. Estes problemas de otimização envolvem 125 equações e 137 
variáveis, sendo 12 variáveis binárias, resultando as três estruturas de RTC apresentadas nas Tabelas 2, 3 e 4.

Tabela 1 - Dados de entrada

\begin{tabular}{cccccc}
\hline Corrente & Período & Tin $(K)$ & Tout $(K)$ & $F(k W / K)$ & $H\left(k W /\left(\mathrm{m}^{2} K\right)\right)$ \\
\hline H1 & 1 & 650 & 370 & 10 & 1 \\
H1 & 2 & 630 & 380 & 10,2 & 1,03 \\
H1 & 3 & 645 & 350 & 10 & 1,01 \\
H2 & 1 & 590 & 370 & 20 & 1 \\
H2 & 2 & 570 & 340 & 20,5 & 1,04 \\
H2 & 3 & 600 & 350 & 20,3 & 1,04 \\
C1 & 1 & 410 & 640 & 15 & 1 \\
C1 & 2 & 390 & 630 & 15 & 1,02 \\
C1 & 3 & 420 & 660 & 14,3 & 1,05 \\
C2 & 1 & 350 & 500 & 13 & 1 \\
C2 & 2 & 340 & 520 & 13,5 & 1,05 \\
C2 & 3 & 320 & 540 & 13 & 1,03 \\
\hline
\end{tabular}

Tabela 2 - Estrutura da RTC para o período 1

\begin{tabular}{llllllll}
\hline Troca & $(i, j, k)$ & $(1,1,1)$ & $(1,2,2)$ & $(2,1,2)$ & $(1, C U, 3)$ & $(2, C U, 3)$ & $(H U, 1,0)$ \\
\hline area $_{i, j, k}$ & $\left(m^{2}\right)$ & 66,0 & 60,1 & 200,7 & 6,9 & 36,3 & 7,3 \\
$q_{i, j, k}$ & $(k W)$ & 600,0 & 1950,0 & 2550,0 & 250,0 & 1850,0 & 300,0 \\
$r h_{i, j, k}$ & & 1,0 & 1,0 & 1,0 & 1,0 & 1,0 & \\
$r c_{i, j, k}$ & & 1,0 & 1,0 & 1,0 & & & 1,0 \\
$F h_{i}$ & $(k W / K)$ & 10,0 & 10,0 & 20,0 & 10,0 & 20,0 & \\
$F c_{j}$ & $(k W / K)$ & 15,0 & 13,0 & 15,0 & & & 15,0 \\
$t h_{i, k}$ & $(K)$ & 650,0 & 590,0 & 590,0 & 395,0 & 462,5 & 680,0 \\
$t h_{i, k+1}$ & $(K)$ & 590,0 & 395,0 & 462,5 & 370,0 & 370,0 & 680,0 \\
$t h s_{i, j, k}$ & $(K)$ & 590,0 & 395,0 & 462,5 & 370,0 & 370,0 & 680,0 \\
$t c_{j, k+1}$ & $(K)$ & 580,0 & 350,0 & 410,0 & 300,0 & 300,0 & 620,0 \\
$t c_{j, k}$ & $(K)$ & 620,0 & 500,0 & 580,0 & 320,0 & 320,0 & 640,0 \\
$t c s_{i, j, k}$ & $(K)$ & 620,0 & 500,0 & 580,0 & 320,0 & 320,0 & 640,0 \\
\hline
\end{tabular}

Tabela 3 - Estrutura da RTC para o período 2

\begin{tabular}{llllllll}
\hline Troca & $(i, j, k)$ & $(1,1,1)$ & $(1,2,2)$ & $(2,1,2)$ & $(2,2,2)$ & $(2, C U, 3)$ & $(H U, 1,0)$ \\
\hline area $_{i, j, k}$ & $\left(m^{2}\right)$ & 66,8 & 83,2 & 236,0 & 22,8 & 49,7 & 8,1 \\
$q_{i, j, k}$ & $(k W)$ & 612,0 & 1938,0 & 2550,0 & 492,0 & 1673,0 & 438,0 \\
$r h_{i, j, k}$ & & 1,0 & 1,0 & 0,878 & 0,122 & 1,0 & \\
$r c_{i, j, k}$ & & 1,0 & 0,798 & 1,0 & 0,202 & & 1,0 \\
$F h_{i}$ & $(k W / K)$ & 10,2 & 10,2 & 20,5 & 20,5 & 20,5 & \\
$F c_{j}$ & $(k W / K)$ & 15,0 & 13,5 & 15,0 & 13,5 & & 15,0 \\
$t h_{i, k}$ & $(K)$ & 630,0 & 570,0 & 570,0 & 570,0 & 421,6 & 680,0 \\
$t h_{i, k+1}$ & $(K)$ & 570,0 & 380,0 & 421,6 & 421,6 & 340,0 & 680,0 \\
$t h s_{i, j, k}$ & $(K)$ & 570,0 & 380,0 & 428,3 & 373,8 & 340,0 & 680,0 \\
$t c_{j, k+1}$ & $(K)$ & 560,0 & 340,0 & 390,0 & 340,0 & 300,0 & 600,8 \\
$t c_{j, k}$ & $(K)$ & 600,8 & 520,0 & 560,0 & 520,0 & 320,0 & 630,0 \\
$t c s_{i, j, k}$ & $(K)$ & 600,8 & 520,0 & 560,0 & 520,0 & 320,0 & 630,0 \\
\hline
\end{tabular}


Tabela 4 - Estrutura da RTC para o período 3

\begin{tabular}{llllllll}
\hline Troca & $(i, j, k)$ & $(1,1,1)$ & $(1,2,2)$ & $(2,1,2)$ & $(2,2,2)$ & $(2, C U, 3)$ & $(H U, 1,0)$ \\
\hline area $_{i, j, k}$ & $\left(m^{2}\right)$ & 55,3 & 113,3 & 224,7 & 6,6 & 50,8 & 17,7 \\
$q_{i, j, k}$ & $(k W)$ & 450,0 & 2500,0 & 2431,0 & 360,0 & 2284,0 & 551,0 \\
$r h_{i, j, k}$ & & 1,0 & 1,0 & 0,840 & 0,160 & 1,0 & \\
$r c_{i, j, k}$ & & 1,0 & 0,874 & 1,0 & 0,126 & & 1,0 \\
$F h_{i}$ & $(k W / K)$ & 10,0 & 10,0 & 20,3 & 20,3 & 20,3 & \\
$F c_{j}$ & $(k W / K)$ & 14,3 & 13,0 & 14,3 & 13,0 & & 14,3 \\
$t h_{i, k}$ & $(K)$ & 645,0 & 600,0 & 600,0 & 600,0 & 462,5 & 680,0 \\
$t h_{i, k+1}$ & $(K)$ & 600,0 & 350,0 & 462,5 & 462,5 & 350,0 & 680,0 \\
$t h s_{i, j, k}$ & $(K)$ & 600,0 & 350,0 & 457,4 & 489,1 & 350,0 & 680,0 \\
$t c_{j, k+1}$ & $(K)$ & 590,0 & 320,0 & 420,0 & 320,0 & 300,0 & 621,5 \\
$t c_{j, k}$ & $(K)$ & 621,5 & 540,0 & 590,0 & 540,0 & 320,0 & 660,0 \\
$t c s_{i, j, k}$ & $(K)$ & 621,5 & 540,0 & 590,0 & 540,0 & 320,0 & 660,0 \\
\hline
\end{tabular}

Com os dados apresentados nas Tabelas 2, 3 e 4, conclui-se que o modelo desenvolvido no presente trabalho é viável nos três períodos de operação. Aplicando-se o procedimento proposto para integrar as estruturas, é identificada a área necessária para cada unidade, a qual é mostrada na Tabela 5. Definindo a duração idêntica nos três períodos de operação, é possível calcular o custo anual com utilidades. A Tabela 6 mostra a comparação entre os custos apresentados por Jiang e Chang (2013) e os custos encontrados pelo modelo simultâneo desenvolvido neste trabalho.

Tabela 5 - Áreas atribuídas

\begin{tabular}{cccc}
\hline Período 1 & Período 2 & Período 3 & Área atribuída $\left(\mathrm{m}^{2}\right)$ \\
\hline 200,7 & 236,0 & 224,7 & 236,0 \\
66,0 & 83,2 & 113,3 & 113,3 \\
60,1 & 66,8 & 55,3 & 66,8 \\
36,3 & 49,7 & 50,8 & 50,8 \\
7,3 & 22,8 & 17,7 & 22,8 \\
6,9 & 8,1 & 6,6 & 8,1 \\
\hline
\end{tabular}

Tabela 6 - Comparação dos custos de capital (CC), operacional (CO) e CTA

\begin{tabular}{lccccc}
\hline & $\begin{array}{c}\mathrm{n}^{\circ} \text { de } \\
\text { unidade }\end{array}$ & $\begin{array}{c}\text { área total } \\
\left(m^{2}\right)\end{array}$ & CC (\$/ano) & CO (\$/ano) & $\begin{array}{c}\text { CTA } \\
(\$ / \mathrm{ano})\end{array}$ \\
\hline $\begin{array}{l}\text { Jiang e Chang (2013) - modelo de } \\
\text { único período }\end{array}$ & 6 & 521,1 & $33.627,0$ & $171.656,3$ & $205.283,2$ \\
\begin{tabular}{l} 
Presente trabalho \\
\hline
\end{tabular} & 6 & 497,8 & $33.210,8$ & $171.656,3$ & $204.867,1$
\end{tabular}

Com os dados apresentados na Tabela 6, observa-se que o modelo desenvolvido, assim como o procedimento para integrar as estruturas, apresentados neste trabalho, alcançam o mesmo custo de utilidades que os custos de Jiang e Chang (2013), porém um custo de capital anualizado menor. Este trabalho apresenta a desvantagem de aumentar um pouco o custo de investimento com a utilização de tubos e válvulas adicionais para auxiliar na integração das estruturas de cada período de operação, porém apresenta uma flexibilidade maior comparado 
com os métodos convencionais, se for necessário um ajuste online na duração dos períodos de operação, pois não é gerado de acordo com as durações pré-determinadas dos períodos, mantendo as taxas de consumo de utilidades em cada período a níveis mínimos.

\section{CONCLUSÕES}

Devido à necessidade industrial de RTC flexíveis que suportam mudanças nas condições de operação em um número finito de períodos, o presente trabalho apresentou um modelo de programação matemática, para alcançar a configuração da rede flexível simultaneamente. Propôs um modelo, o qual é aplicado para cada período de operação separadamente e um algoritmo para integrar as estruturas alcançadas. O modelo realiza o balanço de energia com a temperatura de saída do trocador e tira a relaxação das equações utilizadas para calcular as diferenças de temperaturas dos terminais das unidades de troca térmica. $\mathrm{O}$ estudo de caso realizado resultou em uma RTC com custo de capital menor que os indicados por Jiang e Chang (2013), comprovando a viabilidade do modelo desenvolvido neste trabalho.

\section{REFERÊNCIAS}

Aaltola, J. Simultaneous synthesis of flexible heat exchanger network. Appl. Therm. Eng., v. 22, p. 907-918, 2002.

Chen, C. L.; Kuo, C. H.; Hung, P. S. A Novel Strategy for Synthesis of Flexible HeatExchange Networks. J. Chin. Inst. Chem. Eng., v. 36, n. 5, p. 421-432, 2005.

Chen, J. J. J. Letters to the editors: comments on improvements on a replacement for the logarithmic mean. Chem. Eng. Sci., v. 42, n.10, p. 2488-2489, 1987.

Isafiade, A. J.; Fraser, D. M. Interval based MINLP superstructure synthesis of heat exchanger networks for multi-period operations. Chem. Eng. Res. Des., v. 88, p. 13291341, 2010.

Jiang, D.; Chang, C. T. A New Approach to Genetate Flexible Multiperiod Heat Exchanger Network Designs with Timesharing Mechanisms. Ind. Eng. Chem. Res., v. 52, p. 37943804, 2013.

Ravagnani, M. A. S. S.; Caballero Suárez, J. A. Redes de Cambiadores de Calor. Alicante: Editora de la Universidad de Alicante, 2012.

Verheyen, W.; Zhang, N. Design of flexible heat exchanger network for multi-period operation. Chem. Eng. Sci., v. 61, p. 7730-7753, 2006.

Yee, T. F.; Grossmann, I. E. Simultaneous optimization models for heat integration - II. Heat exchanger network synthesis. Comput. Chem. Eng., v. 14, n. 10, p. 1165-1184, 1990. 